\title{
Low total cholesterol level is the independent predictor of poor outcomes in patients with acute ischemic stroke: a hospital-based prospective study
}

Wenjuan Zhao ${ }^{1,2}$, Zhongping An ${ }^{1,2^{*}}$, Yan Hong ${ }^{1,2}$, Guanen Zhou ${ }^{1,2}$, Jingjing Guo ${ }^{2,3}$, Yongli Zhang ${ }^{2,4}$, Yuanju Yang ${ }^{1,2}$, Xianjia Ning ${ }^{5}$ and Jinghua Wang ${ }^{5}$

\begin{abstract}
Background: Total cholesterol is a well-documented risk factor for coronary disease. Previous studies have shown that high total cholesterol level is associated with better stroke outcomes, but the association of low total cholesterol levels and ischemic stroke outcomes is rare. Therefore, we aimed to assess the association of low total cholesterol levels and stroke outcomes among acute ischemic stroke patients in China.

Methods: This study recruited 6407 atherothrombotic infarction patients from Tianjin, China, between May 2005 and September 2014. All patients were categorized into five groups according to TC level quintiles at admission. Differences in subtypes, severity, risk factors, and outcomes at 3, 12, and 36 months after stroke were compared between these groups.

Results: In total, 1256 (19.6\%) patients had low cholesterol levels, with a higher prevalence in men than in women $(23.7 \%$ vs. $11.2 \%, P<0.001)$. Compared with higher cholesterol levels, the lowest cholesterol level quintile (TC, $<4.07 \mathrm{mmol} / \mathrm{L}$ ) was associated with older age (64.7 years, $P=0.033)$, anterior circulation infarct $(22.8 \%)$, atrial fibrillation (4.9\%), current smoking (41.1\%), and alcohol consumption (21.1\%) and lower frequencies of hypertension (72.9\%), diabetes (30.7\%), and obesity (9.9\%). Dependency and recurrence rates were significantly higher at 36 months in patients in the lowest TC level quintile than in those with higher cholesterol levels (dependency rates, $51.2 \%$ vs $45.2 \% ; P=0.007$ and recurrence rates, $46.3 \%$ vs $37.3 \%, P=0.001$ ). Moreover, these differences remained after adjustment for age, sex, stroke severity, and Oxfordshire Community Stroke Project classification (odds ratios [ORs] for dependency rate, 1.41; $95 \%$ confidence interval [Cl], 1.11, 1.79; $P=0.005$ and recurrence rate, $1.50 ; 95 \% \mathrm{Cl}, 1.19,1.89 ; P=0.001)$. However, mortality rates after stroke were not significantly different between the groups.

Conclusions: These findings suggest that statin treatment for patients with atherothrombotic infarction and low cholesterol levels increase long-term dependency and recurrence rates, but do not increase mortality rates. It is crucial to highlight the different impact of statin treatment on patients with atherothrombotic infarction and lower cholesterol levels for secondary stroke prevention in China.
\end{abstract}

Keywords: Total cholesterol, Atherothrombotic ischemic stroke, Outcomes

\footnotetext{
*Correspondence: tjhhazp@sina.com

'Department of Neurology, Tianjin Huanhu Hospital, 122 Qixiangtai Road, Hexi District, Tianjin 300060, China

${ }^{2}$ Tianjin Key Laboratory of Cerebral Vascular and Neurodegenerative Disease,

Tianjin, China

Full list of author information is available at the end of the article
} 


\section{Background}

In 2010, stroke was the second most common cause of death and the third most common cause of reduced disability-adjusted life-years worldwide. Although agestandardized rates of stroke mortality have decreased worldwide in the past two decades, the absolute numbers of annual stroke cases, stroke survivors, related deaths, and the global burden of stroke disability-adjusted lifeyears are high and are increasing $[1,2]$. However, stroke has recently become the leading cause of death in rural areas, and the third most common cause of death in urban areas in China [3]. High total cholesterol (TC) level is a well-documented risk factor for coronary disease [4, 5], but the association between total cholesterol levels and stroke outcome is unclear. A large numbers of studies indicated that high TC level was associated with better stroke outcomes [6-8], but high TC level was associated with worse outcomes in other studies $[9,10]$. Low TC levels were associated with hemorrhagic, but not ischemic stroke $[11,12]$. The recent incidence of stroke in China has increased dramatically, with economic development [13]; however, a large-scale study of the association between TC level and stroke outcomes is rare in China, especially in patients with atherothrombotic infarction.

Therefore, we aimed to assess the association of low TC level on admission and short-term and long-term stroke outcomes after acute ischemic stroke (AIS) in patients in China.

\section{Methods}

\section{Subjects}

All consecutive patients with first-ever AIS who were admitted to the Stroke Unit in Tianjin Huanhu Hospital within $72 \mathrm{~h}$ of stroke onset between in May 2005 and September 2014 were recruited to this study. A clinical diagnosis of stroke was made according to the World Health Organization criteria and confirmed by neuroimaging (computed tomography/magnetic resonance imaging) [14]. Cases of transient ischemic attack were excluded from this study, and all atherothrombotic infarction patients classified according to the Trial of Org 10,172 in Acute Stroke Treatment (TOAST) for large artery atherothrombotic and small artery occlusion (SAO) were analyzed in this study [15]. All patients were treated using statin, and followed up for less than 3 months after AIS and TC level on admission was available.

The study was approved by the ethics committee for medical research at Tianjin Huanhu Hospital and the Tianjin Health Bureau, and a written informed consent for each participant was obtained during recruitment.

\section{Data collection and group}

The detailed information on ischemic stroke subtype, stroke severity, previous history of diseases, stroke risk factors, laboratory examination results, and outcomes at 3,12 , and 36 months after stroke were collected using a standardized questionnaire.

All patients were categorized into five groups according to the TC level quintile at admission: group 1, $<4.07 \mathrm{mmol} / \mathrm{L}$; group 2, 4.07-4.61 $\mathrm{mmol} / \mathrm{L}$; group 3, $4.62-5.15 \mathrm{mmol} / \mathrm{L}$; group 4, 5.16-5.81 $\mathrm{mmol} / \mathrm{L}$; and group $5,>5.81 \mathrm{mmol} / \mathrm{L}$.

\section{Stroke subtypes}

Stroke subtypes were defined as total anterior circulation infarct (TACI), partial anterior circulation infarct (PACI), posterior circulation infarct (POCI), and lacunar infarct (LACI) according to Oxfordshire Community Stroke Project (OCSP) classification criteria [16].

\section{Neurological function deficit and stroke severity}

Neurological function deficit was defined using the National Institute of Health stroke scale (NIHSS), Barthel index (BI) [17], and modified rankin scale (mRS) on admission [18]. Stroke severity was categorized into 3 groups on the basis of NIHSS score: mild (NIHSS $\leq 7$ ), moderate (NIHSS between 8 to 16 ), and severe (NIHSS $\geq 17$ ) [19].

\section{Risk factors}

Stroke risk factors included a medical history of hypertension (defined as self-reported history of hypertension or using antihypertension drugs), diabetes mellitus (DM, defined as history of DM or using hypoglycemic medications at discharge), atrial fibrillation (AF, defined as history of AF, confirmed by at least one electrocardiogram or the presence of the arrhythmia during hospitalization); and modifiable lifestyle factors, including current smoking status, alcohol consumption, and obesity (body mass index $\geq 30 \mathrm{~kg} / \mathrm{m}^{2}$ ).

\section{Outcome definition}

Stroke outcomes were described on the basis of mortality, recurrence, and dependency rates at the short-term (at 3 months), medium-term (at 12 months), and long-term (at 36 months) after stroke; outcomes were assessed using face-to-face or telephone follow-up. Death was defined as all-cause cumulative death at the corresponding time points after stroke, and this information was collected from patients' family members by telephone follow-up. Recurrence was defined as new-onset vascular events (stroke, myocardial infarction, and venous thrombosis) after 30 days of initial stroke in all survivors patients who were followed up using face-to race interviews or telephone calls. Dependency rate was defined as $\mathrm{mRS}>2$ 
among all survivors followed up using face-to race interview or telephone calls [20].

\section{Follow-up periods}

Follow-up was conducted according to a predetermined procedure; the trained neurologists reexamined patients in the outpatient department at 3, 12, 24, and 36 months after stroke. All patients were followed-up by face-toface interview, but those patients reexamined in the local hospital were followed-up by telephone.

\section{Statistical analysis}

Age is presented as mean (standard deviation), and NIHSS, BI, and mRS are presented as median (interquartile range). These continuous variables were compared between the five TC groups using one-way analysis of variance (ANOVA) or Kruskal-Wallis tests. At the different time periods after stroke, categorical variables, including stroke subtype, stroke severity, risk factors, and outcomes, are presented as number (percentage), and the trends were compared using Chi-square tests. Differences in outcomes were compared between the five groups using univariate and multivariate logistic regression models and are presented as unadjusted and adjusted (by sex, age, stroke severity, OCSP, and risk factors [hypertension, diabetes, atrial fibrillation, artery stenosis, obesity, current smoking, and alcohol consumption]) odds ratios (OR), respectively, with $95 \%$ confidence intervals (CI). All statistical analyses were performed using SPSS version 15.0 (SPSS Inc., Chicago, IL), and two-tailed $P$ values $<0.05$ were considered statistically significant.

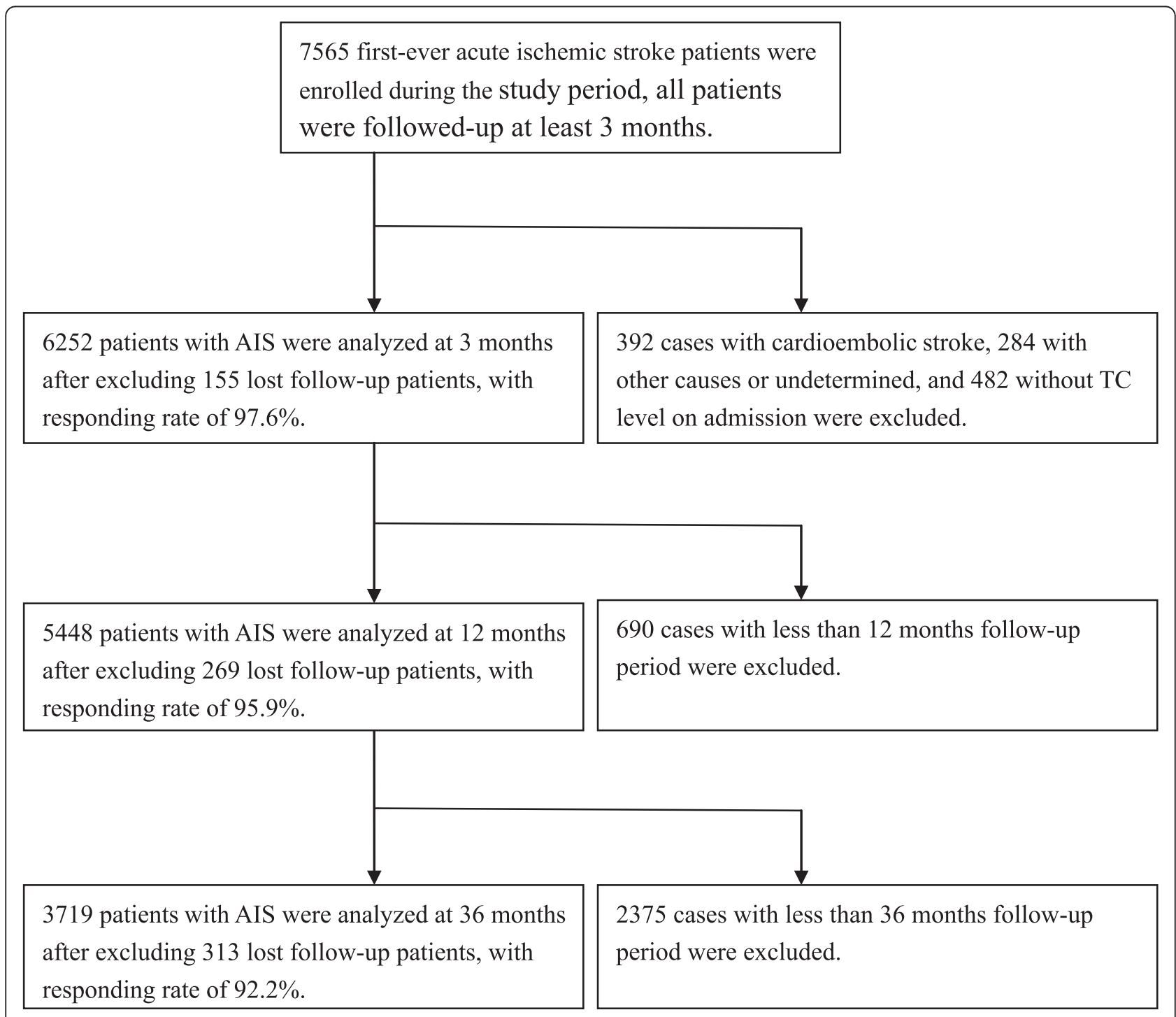

Fig. 1 Flow diagram of participants 


\section{Results}

Of the 7565 AIS patients recruited between May 2005 and September 2014, 392 patients with cardioembolic stroke, 284 patients with other and undetermined causes and 482 patients without a TC level recorded at admission were excluded, resulting in 6407 patients with atherothrombotic infarction that were included. At 3 months, 155 patients were lost to follow-up, resulting in 6252 patients (response rate, 97.6\%). At 12 months, 269 patients were lost to follow-up, resulting in 5448 patients (response rate, $95.3 \%$ ). At 36 months, 313 patients were lost to follow-up, resulting in 3719 patients (response rate, $92.2 \%$ ) (Fig. 1).

The lowest TC levels were present in 1256 (19.6\%) patients (1022 [23.7 \%] men; 234 [11.2 \%] women; $P<0.001$; Table 1). The mean age at stroke onset decreased with increasing TC levels $(P=0.033)$. The prevalence of TACI decreased, and the prevalence of POCI increased, with increasing TC levels $(P<0.001)$. Moreover, the proportion of patients with severe stroke increased with increasing TC levels $(P<0.05)$; neurological function deficits at admission were worse with increasing TC levels $(P<0.05)$.

The prevalence of AF, current smoking, and alcohol consumption were significantly lower with higher TC levels (all $P<0.05$ ). In contrast, the prevalence of hypertension, diabetes, and obesity increased with increasing
TC levels (all $P<0.001$ ). The prevalence of artery stenosis was not significantly different (Table 2).

There were no obvious differences in mortality and dependency rates at all time points after AIS between the TC level groups (Table 3). However, the recurrence rate at 3 months was remarkably higher with higher TC levels (group 1, $7.0 \%$; group 2, $7.4 \%$; group 3, $9.8 \%$; group 4, $9.7 \%$; and group 5, $8.6 \% ; P=0.038$ ). The trend in recurrence rates at 36 months after stroke was the opposite to that at 3 months (group 1, $46.3 \%$; group 2, $41.0 \%$; group 3, $41.1 \%$; group 4, $37.7 \%$; and group 5, $37.3 \% ; P=0.001)$.

In the univariate analysis, compared with the lowest TC levels (group 1), mortality rates were lower at 3 and 12 months after stroke (by 32 and $29 \%$, respectively), and the recurrence rate was lower at 36 months after stroke (by $20 \%$ ) in those with TC levels of 4.62 $5.15 \mathrm{mmol} / \mathrm{L}$ (group 3). Compared with the lowest TC level group (group 1), the dependency rate was $21 \%$ lower in group 2, $25 \%$ lower in group 3, 20 \% lower in group 4, and $21 \%$ lower in group 5 (Table 4).

In the multivariate analysis, the dependency rate was significantly lower in the higher TC level groups compared with group 1, by $21 \%$ in group 2, $24 \%$ in group $3,22 \%$ in group 4 , and $29 \%$ in group 5 . The recurrence rates in TC level groups 3 and 5 were significantly lower

Table 1 The clinical characteristics and risk factors in patients with acute ischemic stroke by TC levels

\begin{tabular}{|c|c|c|c|c|c|c|}
\hline Characteristics & Group 1 & Group 2 & Group 3 & Group 4 & Group 5 & $P$ \\
\hline Numbers, $n(\%)$ & 1256 (19.6) & $1282(20.0)$ & $1283(20.0)$ & $1312(20.5)$ & $1274(19.9)$ & - \\
\hline Gender, n (\%) & & & & & & $<0.001$ \\
\hline Men & $1022(23.7)$ & $959(22.2)$ & $907(21.0)$ & $794(18.4)$ & $637(14.7)$ & \\
\hline Women & $234(11.2)$ & $323(15.5)$ & $376(18.0)$ & $518(24.8)$ & $637(30.5)$ & \\
\hline Age, year, means (SD) & $64.69(11.55)$ & $64.10(11.46)$ & $63.59(11.43)$ & $63.36(10.98)$ & $63.94(11.12)$ & 0.033 \\
\hline OCSP classification, $n(\%)$ : & & & & & & $<0.001$ \\
\hline $\mathrm{TACl}$ & $63(22.8)$ & $59(21.4)$ & $54(19.6)$ & $46(16.7)$ & $54(19.6)$ & \\
\hline $\mathrm{PACl}$ & $720(20.7)$ & $688(19.8)$ & $704(20.2)$ & $716(20.6)$ & $651(18.7)$ & \\
\hline $\mathrm{LACl}$ & $82(18.2)$ & $118(26.2)$ & $85(18.8)$ & $90(20.0)$ & $76(16.9)$ & \\
\hline $\mathrm{POCl}$ & $391(17.8)$ & $417(18.9)$ & $440(20.0)$ & $460(20.9)$ & $493(22.4)$ & \\
\hline Stroke severity: & & & & & & 0.001 \\
\hline Mild & $849(67.6)$ & $907(70.7)$ & $886(69.1)$ & $889(67.8)$ & $803(63.0)$ & \\
\hline Moderate & $309(24.6)$ & $282(22.0)$ & $311(24.2)$ & $320(24.4)$ & $337(26.5)$ & \\
\hline Severe & $98(7.8)$ & $93(7.3)$ & $86(6.7)$ & $102(7.8)$ & $134(10.5)$ & \\
\hline \multicolumn{7}{|c|}{ Neurological function deficit: } \\
\hline NIHSS & $5(7)$ & $5(6)$ & $5(7)$ & $5(7)$ & $5(7)$ & 0.022 \\
\hline $\mathrm{Bl}$ & $60(45)$ & $60(45)$ & $60(45)$ & $60(45)$ & $60(55)$ & 0.006 \\
\hline $\mathrm{mRS}$ & $3(2)$ & $3(2)$ & $3(2)$ & $3(2)$ & $3(2)$ & 0.006 \\
\hline
\end{tabular}

TC total cholesterol, OCSP Oxfordshire Community Stroke Project, TACl total anterior circulation infarct, $P A C l$ partial anterior circulation infarct, $P O C l$ posterior circulation infarct, $L A C l$ lacunar infarct, NIHSS National Institute of Health stroke scale, BI Barthel index, mRS modified rankin scale 
Table 2 The Prevalence of risk factors in patients with acute ischemic stroke by TC levels

\begin{tabular}{|c|c|c|c|c|c|c|c|}
\hline Risk factors & Total & Group 1 & Group 2 & Group 3 & Group 4 & Group 5 & $P$ \\
\hline Hypertension & $4756(74.2)$ & $916(72.9)$ & $922(71.9)$ & $933(72.7)$ & $987(75.2)$ & $998(78.3)$ & $<0.001$ \\
\hline Diabetes & $2090(32.6)$ & $386(30.7)$ & $370(28.9)$ & $418(32.6)$ & $411(31.3)$ & 505 (39.6) & $<0.001$ \\
\hline Atrial fibrillation & 231 (3.6) & $62(4.9)$ & $46(3.6)$ & $41(3.2)$ & $46(3.5)$ & $36(2.8)$ & 0.010 \\
\hline Artery stenosis & $1572(24.5)$ & $339(27.0)$ & $297(23.2)$ & $295(23.0)$ & $317(24.2)$ & $324(25.4)$ & 0.589 \\
\hline Obesity & $795(12.4)$ & $124(9.9)$ & $141(11.0)$ & $130(10.1)$ & $192(14.6)$ & $208(16.3)$ & $<0.001$ \\
\hline Current smoking & $2565(40.0)$ & $516(41.1)$ & $575(44.9)$ & $524(40.8)$ & $511(38.9)$ & $439(34.5)$ & $<0.001$ \\
\hline Alcohol consumption & $1243(19.4)$ & $265(21.1)$ & 252 (19.7) & $253(19.7)$ & $247(18.8)$ & $226(17.7)$ & 0.032 \\
\hline
\end{tabular}

TC total cholesterol

(by 20 and $27 \%$, respectively) than that in the lowest TC group (group 1; Table 5).

\section{Discussion}

In this single-center study using a large stroke registry in Tianjin, China, we assessed differences in age, sex, stroke subtype, stroke severity, prevalence of risk factors, and stroke outcomes between patients with atherothrombotic infarction with and without low TC levels. As a result, a low TC level was an independent risk factor for outcomes in patients with atherothrombotic infarction.

A high cholesterol level is a powerful risk factor for coronary heart disease, but its role in stroke remains controversial. Although observational studies have not found a clear association between cholesterol levels and stroke [21-25], associations between high serum TC levels and an increased risk of ischemic stroke have been reported [26, 27]. A study in Americans indicated a positive association of TC levels with atherothrombotic infarction [28]. Only one study conducted in Japan reported that serum TC levels were positively associated with an increased age-adjusted risk of atherothrombotic infarction in Japanese women aged $\geq 40$ years, but not in men [29].
A recent study showed that serum cholesterol $<160 \mathrm{mg} / \mathrm{dL}$ was a risk factor for hemorrhagic stroke and increased the risk of death after hemorrhagic stroke, but a reverse trend was found for ischemic stroke incidence and death $[11,12]$. Moreover, a study conducted in Japan indicated that a higher TC level increased the risk of cerebral infarction [11]. In a hospital-based study, low serum TC levels were associated with increased risk of severe stroke, TACI, and poor functional outcomes in patients with ischemic stroke who had received pre-stroke statin treatment, and the short-term and long-term mortality rates were significantly higher in patients with low cholesterol levels [30]. Another community-based cohort study indicated that low cholesterol levels significantly increased the risks of stroke and heart disease [31]. In contrast with these previous findings, there was no significant association between low cholesterol levels and mortality after stroke in the present study.

A meta-analysis including 450,000 individuals from 45 observational cohorts suggested that there was no association between TC level and the risk of fatal stroke during an average follow-up of 16 years [32]. In another meta-analysis of approximately 1 million individuals, a clear association was found between the serum TC level

Table 3 The outcome at 3, 12, and 36 months after stroke in acute ischemic stroke patients by TC levels

\begin{tabular}{|c|c|c|c|c|c|c|c|}
\hline Outcomes & Total & Group 1 & Group 2 & Group 3 & Group 4 & Group 5 & $P$ \\
\hline \multicolumn{8}{|l|}{3 months: } \\
\hline Mortality & $340(5.4)$ & $76(6.2)$ & 57 (4.6) & $54(4.3)$ & $73(5.7)$ & $80(6.5)$ & 0.414 \\
\hline Dependency & $602(10.1)$ & $124(10.7)$ & 125 (10.3) & $108(8.9)$ & $111(9.1)$ & $134(11.4)$ & 0.962 \\
\hline Recurrence & 207 (8.5) & $81(7.0)$ & $90(7.4)$ & $118(9.8)$ & $118(9.7)$ & $100(8.6)$ & 0.038 \\
\hline \multicolumn{8}{|l|}{12 months: } \\
\hline Mortality & $454(8.3)$ & $91(8.6)$ & 87 (7.9) & $68(6.3)$ & $94(8.4)$ & $114(10.6)$ & 0.080 \\
\hline Dependency & 1218 (24.4) & 258 (26.6) & 239 (23.4) & $234(22.9)$ & $250(24.4)$ & $237(24.7)$ & 0.538 \\
\hline Recurrence & 1153 (22.7) & $229(23.3)$ & 219 (20.9) & $261(25.4)$ & $229(22.0)$ & $215(21.8)$ & 0.647 \\
\hline \multicolumn{8}{|l|}{36 months: } \\
\hline Mortality & $588(15.8)$ & 109 (15.5) & $127(16.1)$ & 103 (13.6) & 117 (15.9) & $132(18.2)$ & 0.229 \\
\hline Dependency & 1447 (46.2) & $305(51.2)$ & 301 (45.4) & $290(44.1)$ & $282(45.5)$ & $269(45.2)$ & 0.070 \\
\hline Recurrence & 1359 (40.7) & $294(46.3)$ & $291(41.0)$ & $289(41.1)$ & 249 (37.7) & 236 (37.3) & 0.001 \\
\hline
\end{tabular}

TC total cholesterol, OR odds ratios, $\mathrm{Cl}$ confidence intervals 
Table 4 Un-adjusted OR (95\% Cl) of TC levels in the outcomes at 3, 12, and 36 months after stroke in acute ischemic stroke patients

\begin{tabular}{|c|c|c|c|c|c|}
\hline Outcomes & Group 1 & Group 2 & Group 3 & Group 4 & Group 5 \\
\hline \multicolumn{6}{|l|}{3 months: } \\
\hline Mortality & 1.00 & $0.72(0.51,1.03)$ & $0.68(0.47,0.97)^{a}$ & $0.91(0.66,1.27)$ & $1.04(0.75,1.44)$ \\
\hline Dependency & 1.00 & $0.96(0.74,1.24)$ & $0.81(0.62,1.07)$ & $0.83(0.64,1.09)$ & $1.07(0.83,1.39)$ \\
\hline Recurrence & 1.00 & $0.87(0.66,1.14)$ & $0.80(0.60,1.05)$ & $0.79(0.60,1.05)$ & $1.02(0.78,1.33)$ \\
\hline \multicolumn{6}{|l|}{12 months: } \\
\hline Mortality & 1.00 & $0.91(0.67,1.23)$ & $0.71(0.51,0.99)^{\mathrm{a}}$ & $0.98(0.72,1.32)$ & $1.27(0.95,1.70)$ \\
\hline Dependency & 1.00 & $0.84(0.69,1.03)$ & $0.82(0.67,1.01)$ & $0.89(0.73,1.09)$ & $0.91(0.74,1.11)$ \\
\hline Recurrence & 1.00 & $0.90(0.74,1.09)$ & $0.85(0.70,1.04)$ & $0.93(0.76,1.13)$ & $0.94(0.77,1.15)$ \\
\hline \multicolumn{6}{|l|}{36 months: } \\
\hline Mortality & 1.00 & $1.05(0.79,1.38)$ & $0.86(0.64,1.15)$ & $1.03(0.78,1.37)$ & $1.21(0.92,1.60)$ \\
\hline Dependency & 1.00 & $0.79(0.64,0.99)^{a}$ & $0.75(0.60,0.94)^{a}$ & $0.80(0.64,0.99)^{a}$ & $0.79(0.63,0.99)^{a}$ \\
\hline Recurrence & 1.00 & $0.87(0.70,1.07)$ & $0.80(0.64,0.99)^{\mathrm{a}}$ & $0.83(0.67,1.03)$ & $0.82(0.66,1.01)$ \\
\hline
\end{tabular}

TC total cholesterol, $O R$ odds ratios, $\mathrm{Cl}$ confidence intervals

${ }^{\mathrm{a}}$ indicated $\mathrm{P}<0.05$ refence as Group 1

and the risk of fatal myocardial infarction, but again, there was no obvious association with fatal stroke [25].

Consistent with the findings of previous studies, the present study found that, compared with patients with higher TC levels, patients with low TC levels were more likely to be male; older; to have TACI or AF; be a current smoker; and currently consume alcohol. Regarding age, the TC level was associated with fatal ischemic stroke in patients aged $<70$ years, but not in patients aged $>70$ years in a previous study [25]. Moreover, patients in the lowest TC level group were less likely to have hypertension, diabetes, and obesity than patients in the higher TC level groups.

Clinical trials using statins to reduce cholesterol levels in patients with cardiovascular or cerebrovascular diseases have shown significant reductions in the risk of stroke [33-39] and that reduced cholesterol levels can reduce the incidence of stroke in high-risk populations and in patients with a stroke or transient ischemic attack [25, 32]. Statin therapy has become the most important advancement in stroke prevention since aspirin and blood pressure-lowering therapies were introduced. Statins not only lower the overall risk of stroke but also reduce the progression of carotid atherosclerosis; reduce inflammation and endothelial dysfunction; decrease platelet aggregation to improve fibrinolysis; lower blood pressure; and decrease the risk of thromboembolic complications in the brain by reducing the incidence of myocardial infarction. Statins might also have a neuroprotective effect [40-42]. However, clinicians should explore the effects of statin

Table 5 Adjusted $^{\mathrm{a}}$ OR (95\% Cl) of TC levels in the outcomes at 3, 12, and 36 months after stroke in acute ischemic stroke patients

\begin{tabular}{|c|c|c|c|c|c|}
\hline Outcomes & Group 1 & Group 2 & Group 3 & Group 4 & Group 5 \\
\hline \multicolumn{6}{|l|}{3 months: } \\
\hline Mortality & 1.00 & $0.77(0.52,1.13)$ & $0.73(0.49,1.08)$ & $1.08(0.74,1.55)$ & $0.99(0.68,1.44)$ \\
\hline Dependency & 1.00 & $0.98(0.75,1.29)$ & $0.84(0.63,1.10)$ & $0.88(0.67,1.17)$ & $1.04(0.79,1.37)$ \\
\hline Recurrence & 1.00 & $0.89(0.68,1.18)$ & $0.82(0.62,1.09)$ & $0.85(0.64,1.13)$ & $1.02(0.77,1.35)$ \\
\hline \multicolumn{6}{|l|}{12 months: } \\
\hline Mortality & 1.00 & $0.99(0.70,1.39)$ & $0.74(0.52,1.07)$ & $1.11(0.79,1.57)$ & $1.22(0.87,1.71)$ \\
\hline Dependency & 1.00 & $0.86(0.70,1.06)$ & $0.83(0.68,1.03)$ & $0.92(0.75,1.13)$ & $0.87(0.71,1.09)$ \\
\hline Recurrence & 1.00 & $0.91(0.74,1.11)$ & $0.86(0.70,1.05)$ & $0.95(0.77,1.16)$ & $0.89(0.72,1.10)$ \\
\hline \multicolumn{6}{|l|}{36 months: } \\
\hline Mortality & 1.00 & $1.15(0.84,1.58)$ & $0.88(0.63,1.23)$ & $1.12(0.81,1.56)$ & $1.07(0.77,1.49)$ \\
\hline Dependency & 1.00 & $0.79(0.63,0.99)^{\mathrm{a}}$ & $0.76(0.60,0.95)^{\mathrm{a}}$ & $0.78(0.62,0.99)^{\mathrm{a}}$ & $0.71(0.56,0.90)^{a}$ \\
\hline Recurrence & 1.00 & $0.86(0.70,1.07)$ & $0.80(0.64,0.99)^{\mathrm{a}}$ & $0.81(0.64,1.01)$ & $0.73(0.58,0.91)^{a}$ \\
\hline
\end{tabular}

TC total cholesterol, OR odds ratios, $\mathrm{Cl}$ confidence intervals

${ }^{a}$ Adjusted by sex, age, stroke severity, OCSP, risk factors (hypertension, diabetes, atrial fibrillation, artery stenosis, obesity, current smoking, and alcohol consumption) as covariates 
treatment in patients with atherothrombotic infarction and low TC levels.

Given the higher dependency and recurrence rates at 36 months after stroke in patients with the lowest TC levels at admission in the present study, the benefits of statin treatment for atherothrombotic infarction did not appear to occur, supporting the role of low TC levels as an independent risk factor for outcomes in these patients. The mechanism explaining the association between low TC levels and poorer stroke outcomes is unknown. However, differences in race and social development between Asian and Western populations might partially explain the relationship.

There are several limitations in this study. First, all patients were from a local neurological hospital in Tianjin, China, and may not represent all stroke patients in China. Second, the data on TC levels before stroke was lacking; this may have affected the evaluation of statin treatment.

\section{Conclusion}

In this large, hospital-based, prospective study using a stroke registry from Tianjin, China, we assessed differences in stroke subtype, severity, risk factors, and outcomes at 3,12, and 36 months after stroke in patients with atherothrombotic infarction based on different TC levels. Men and older patients were more likely to have low TC levels, and the frequencies of TACI, AF, current smoking, and alcohol consumption were higher in patients with low TC levels than in patients with higher TC levels. Furthermore, low TC levels were associated with poor long-term outcomes, and a significant negative relationship between cholesterol levels and dependency and recurrence rates at 36 months after stroke onset were observed. However, there was no significant difference in mortality after stroke. Therefore, the TC level appears to be a determinant of long-term outcomes in patients with atherothrombotic infarction; statin treatment in the patients with lower TC levels increased the long-term risk of dependency and recurrence, but not the risk of all-cause death. Therefore, to improve secondary stroke prevention in China, it is important to determine the different impact of statin treatment in patients with atherothrombotic infarction based on cholesterol levels.

\footnotetext{
Abbreviations

AF: atrial fibrillation; AIS: acute ischemic stroke; BI: bethel index Cl: confidence intervals; DM: diabetes mellitus; FG: fasting glucose; HbA1c: glycosylated hemoglobin; HDL-C: high-density lipoprotein cholesterol; LACl: lacunar infarct; LDL-C: low-density lipoprotein cholesterol; mRS: modified rank scale; NIHSS: National Institute of Health stroke scale; OCSP: Oxfordshire Community Stroke Project; PACl: partial anterior circulation infarct; POCl: posterior circulation infarct; RR: relative risk; SAO: small artery occlusion; TACl: total anterior circulation infarct; TC: total cholesterol; TG: triglyceride; TOAST: Trial of Org 10172 in Acute Stroke Treatment.
}

\section{Competing interests}

The authors declare that they have no competing interests.

\section{Authors' contributions}

We thank all participants in this study. WZ was involved in design of the study, data collection, interpretation of the data and writing the manuscript. ZA took part in design of the study, funding obtained, data collection, interpretation of the data and revising the manuscript. $Y H, G Z, J G, Y Z$, and YY were involved in data collection. XN and JW took part in data analysis and revising the manuscript. All authors read and approved the final manuscript.

\section{Acknowledgments}

This study was funded partly by Tianjin Health Bureau of Science and Technology Fund Key Projects (contract: KY12, and 2013KG120).

\section{Author details}

'Department of Neurology, Tianjin Huanhu Hospital, 122 Qixiangtai Road, Hexi District, Tianjin 300060, China. ${ }^{2}$ Tianjin Key Laboratory of Cerebral Vascular and Neurodegenerative Disease, Tianjin, China. ${ }^{3}$ Department of Radiology, Tianjin Huanhu Hospital, Tianjin, China. ${ }^{4}$ Department of Clinical Pharmacy, Tianjin Huanhu Hospital, Tianjin, China. ${ }^{5}$ Department of Epidemiology, Tianjin Neurological Institute \& Department of Neurology, Tianjin Medical University General Hospital, Tianjin, China.

Received: 14 October 2015 Accepted: 10 March 2016

Published online: 15 March 2016

\section{References}

1. Lozano R, Naghavi M, Foreman K, et al. Global and regional mortality from 235 causes of death for 20 age groups in 1990 and 2010: a systematic analysis for the Global Burden of Disease Study 2010. Lancet. 2012;380:2095-128.

2. Murray CJ, Vos T, Lozano R, et al. Disability-adjusted life-years (DALYs) for 291 diseases and injuries in 21 regions, 1990-2010: a systematic analysis for the Global Burden of Disease Study 2010. Lancet. 2012;380:2197-223.

3. The Ministry of Health of the People's Republic of China. China Health Statistics yearbook 2011. Beijing: China Union Medical University Press; 2011.

4. Castelli WP, Anderson K, Wilson PW, et al. Lipids and coronary heart disease: the Framingham Study. Ann Epidemiol. 1992;2:23-8.

5. Nam B-H, Kannel W, D'Agostino RB. Search for an optimal atherogenic lipid risk profile: from The Framingham Study. Am J Cardiol. 2006;97:372-5.

6. Markaki I, Nilsson U, Kostulas K, et al. High Cholesterol Levels Are Associated with Improved Long-term Survival after Acute Ischemic Stroke. J Stroke Cerebrovasc. 2014;23:e47-53.

7. Pan SL, Lien IN, Chen TH. Is higher serum total cholesterol level associated with better long-term functional outcomes after noncardioembolic ischemic stroke? Arch Phys Med Rehabil. 2010;91:913-8.

8. Vauthey C, de Freitas GR, van Melle G, et al. Better outcome after stroke with higher serum cholesterol levels. Neurology. 2000;54:1944-9.

9. von Budingen $\mathrm{HC}$, Baumgartner RW, Baumann $\mathrm{CR}$, et al. Serum cholesterol levels do not influence outcome or recovery in acute ischemic stroke. Neurol Res. 2008;30:82-4.

10. Cui R, Iso H, Yamagishi $K$, et al. High serum total cholesterol levels is a risk factor of ischemic stroke for general Japanese population: The JPHC study. Atherosclerosis. 2012;221:565-9.

11. Suzuki K, Izumi M, Sakamoto T, et al. Blood pressure and total cholesterol level are critical risks especially for hemorrhagic stroke in Akita, Japan. Cerebrovasc Dis. 2011;31:100-6.

12. Iso H, Jacobs Jr DR, Wentworth D, et al. Serum cholesterol levels and six-year mortality from stroke in 350,977 men screened for the Multiple Risk Factor Intervention Trial. N Engl J Med. 1989;320:904-10.

13. Wang J, Ning $X$, Yang $L$, et al. Sex Differences in Trends of Incidence and Mortality of First-Ever Stroke in Rural Tianjin, China from 1992 to 2012. Stroke. 2014;45(6):1626-31.

14. World Health Organization Task Force on Stroke and Other Cerebrovascular Disorders. Stroke-1989. Recommendations on stroke prevention, diagnosis, and therapy. Report of the WHO task force on stroke and other cerebrovascular disorders. Stroke. 1989;20(10):1407-31. 
15. Adams HP, Bendixen BH, Kappelle $L$, et al. Classification of subtype of acute ischemic stroke: definitions for use in a multicenter clinical trial: TOAST: Trial of Org 10172 in Acute Stroke Treatment. Stroke. 1993;24:35-41.

16. Bamford J, Sandercock $P$, Dennis $M$, et al. Classification and natural history of clinically identifiable subtypes of cerebral infarction. Lancet. 1991;337:1521-6.

17. Barthel DW, Mahoney FI. Functional evaluation: the barthel index. Md State Med J. 1965;14:61-5.

18. de Haan R, Limburg M, Bossuyt P, van der Meulen J, Aaronson N. The clinical meaning of Rankin 'handicap' grades after stroke. Stroke. 1995;26:2027-30.

19. Kim J-S, Lee K-B, Roh H, et al. Gender Differences in the Functional Recovery after Acute Stroke. J Clin Neurol. 2010;6:183-8.

20. Banks JL, Marotta CA. Outcomes validity and reliability of the modified Rankin scale: implications for stroke clinical trials: a literature review and synthesis. Stroke. 2007;38(3):1091-6.

21. Horenstein RB, Smith DE, Mosca L. Cholesterol predicts stroke mortality in the Women's Pooling Project. Stroke. 2002;33:1863-8.

22. Kurth $T$, Everett BM, Buring JE, et al. Lipid levels and the risk of ischemic stroke in women. Neurology. 2007;68:556-62.

23. Prospective Studies Collaboration, Lewington S, Whitlock G, et al. Blood cholesterol and vascular mortality by age, sex, and blood pressure: a meta-analysis of individual data from 61 prospective studies with 55000 vascular deaths. Lancet. 2007;370:1829-39.

24. Harmsen $P$, Lappas $G$, Rosengren A, et al. Longterm risk factors for stroke. Twenty-eight years of follow-up of 7457 middle-aged men in Goteborg, Sweden. Stroke. 2006;37:1663-7.

25. Wattanakit K, Folsom AR, Chambless LE, et al. Risk factors for cardiovascular event recurrence in the Atherosclerosis Risk in Communities (ARIC) study. Am Heart J. 2005;149:606-12.

26. Lindenstrom E, Boysen G, Nyboe J. Influence of total cholesterol, high density lipoprotein cholesterol, and triglycerides on risk of cerebrovascular disease: the Copenhagen City Heart Study. Br Med J. 1994;309:11-5.

27. Benfante $R$, Yano $K$, Hwang $L$, et al. Elevated serum cholesterol is a risk factor for both coronary heart disease and thromboembolic stroke in Hawaiian Japanese men. Stroke. 1994;25:814-20.

28. Ohira T, Shahar E, Chambless LE, et al. Risk factors for ischemic stroke subtypes: the atherosclerosis risk in communities study. Stroke. 2006;37:2493-8

29. Tanizaki Y, Kiyohara Y, Kato I, et al. Incidence and risk factors for subtypes of cerebral infarction in a general population: the Hisayama study. Stroke. 2000;31:2616-22.

30. Koton S, Molshatzki N, Bornstein NM, et al. Low cholesterol, statins and outcomes in patients with first-ever acute ischemic stroke. Cerebrovasc Dis. 2012;34(3):213-20.

31. Nago N, Ishikawa S, Goto T, et al. Low cholesterol is associated with mortality from stroke, heart disease, and cancer: the Jichi Medical Schoo Cohort Study. J Epidemiol. 2011;21(1):67-74.

32. Prospective studies collaboration. Cholesterol, diastolic blood pressure, and stroke: 13,000 strokes in 450,000 people in 45 prospective cohorts. Lancet. 1995;346:1647-53.

33. White HD, Simes J, Anderson NE, et al. Pravastatin therapy and the risk of stroke. N Engl J Med. 2000;343:317-26.

34. Schwartz GG, Olsson AG, Ezekowitz MD, et al. Effects of atorvastatin on early recurrent ischemic events in acute coronary syndromes. The MIRACL study: a randomized controlled trial. JAMA. 2001;285:1711-8.

35. LaRosa JC, Grundy SM, Waters DD, et al. Intensive lipid lowering with atorvastatin in patients with stable coronary disease. N Engl J Med. 2005;352:1425-35

36. Colhoun HM, Betteridge DJ, Durrington PN, et al. Primary prevention of cardiovascular disease with atorvastatin in type 2 diabetes in the Collaborative Atorvastatin Diabetes Study (CARDS): multicentre randomized placebocontrolled trial. Lancet. 2004;364:685-96.

37. Sever PS, Dahlof B, Poulter NR, et al. Prevention of coronary and stroke events with atorvastatin in hypertensive patients who have average or lower-than-average cholesterol concentrations, in the Anglo-Scandinavian Cardiac Outcomes Trial-Lipid Lowering Arm (ASCOT-LLA): A multicentre randomised controlled trial. Lancet. 2003;361:1149-58.

38. Heart Protection Study Collaborative Group. MRC/BHF Heart Protection Study of cholesterol lowering with simvastatin in 20536 high-risk individuals: a randomized placebo-controlled trial. Lancet. 2002;360:7-22.
39. Amarenco P, Bogousslavsky J, Callahan III A, et al. Highdose atorvastatin after stroke or transient ischemic attack. N Engl J Med. 2006:355:549-59.

40. Amarenco P, Lavallee P, Touboul P-J. Stroke prevention, blood cholesterol, and statins. Lancet Neurol. 2004:3:271-8.

41. Amarenco $P$, Moskowitz MA. The dynamics of statins: from event prevention to neuroprotection. Stroke. 2006;37:294-6.

42. Lavallée PC, Labreuche J, Gongora-Rivera F, et al. Placebo-controlled trial of high-dose atorvastatin in patients with severe cerebral small vessel disease. Stroke. 2009:40(5):1721-8.

\section{Submit your next manuscript to BioMed Central and we will help you at every step:}

- We accept pre-submission inquiries

- Our selector tool helps you to find the most relevant journal

- We provide round the clock customer support

- Convenient online submission

- Thorough peer review

- Inclusion in PubMed and all major indexing services

- Maximum visibility for your research

Submit your manuscript at www.biomedcentral.com/submit
Biomed Central 